\title{
BMJ open Effectiveness and cost-effectiveness of a universal parenting skills programme in deprived communities: multicentre randomised controlled trial
}

\author{
D E Simkiss, ${ }^{1}$ H A Snooks, ${ }^{2}$ N Stallard, ${ }^{1}$ P K Kimani, ${ }^{1}$ B Sewell, ${ }^{3}$ D Fitzsimmons, ${ }^{3}$ \\ R Anthony, ${ }^{2}$ S Winstanley, ${ }^{2}$ L Wilson, ${ }^{2}$ C J Phillips, ${ }^{3}$ S Stewart-Brown ${ }^{1}$
}

To cite: Simkiss DE, Snooks HA, Stallard N, et al. Effectiveness and costeffectiveness of a universal parenting skills programme in deprived communities: multicentre randomised controlled trial. BMJ Open 2013;3:e002851. doi:10.1136/ bmjopen-2013-002851

- Prepublication history and additional material for this paper is available online. To view these files please visit the journal online (http://dx.doi.org/10.1136/ bmjopen-2013-002851).

Received 14 March 2013 Revised 12 June 2013 Accepted 24 June 2013

${ }^{1}$ Health Sciences Research Institute, Warwick Medical School, University of Warwick, Coventry, UK ${ }^{2}$ Centre for Health Information, Research and Evaluation, Institute of Life Science, College of Medicine, Swansea University, Swansea, Wales, UK ${ }^{3}$ Swansea Centre for Health Economics, College of Human and Health Sciences, Swansea University, Singleton Park, SA2 8PP,

Swansea University,

Swansea, Wales, UK

Correspondence to Dr D E Simkiss;

d.e.simkiss@warwick.ac.uk

\section{ABSTRACT}

Objective: To evaluate the effectiveness and cost utility of a universally provided early years parenting programme.

Design: Multicentre randomised controlled trial with cost-effectiveness analysis.

Setting: Early years centres in four deprived areas of South Wales.

Participants: Families with children aged between 2 and 4 years. 286 families were recruited and randomly allocated to the intervention or waiting list control.

Intervention: The Family Links Nurturing Programme (FLNP), a 10-week course with weekly $2 \mathrm{~h}$ facilitated group sessions.

Main outcome measures: Negative and supportive parenting, child and parental well-being and costs assessed before the intervention, following the course (3 months) and at 9 months using standardised measures.

Results: There were no significant differences in primary or secondary outcomes between trial arms at 3 or 9 months. With ' + ' indicating improvement, difference in change in negative parenting score at 9 months was $+0.90(95 \% \mathrm{Cl}-1.90$ to 3.69$)$; in supportive parenting, $+0.17(95 \% \mathrm{Cl}-0.61$ to 0.94$)$; and 12 of the 17 secondary outcomes showed a non-significant positive effect in the FLNP arm. Based on changes in parental well-being (SF-12), the cost per quality-adjusted life year (QALY) gained was estimated to be $£ 34913$ (range $21485-46578$ ) over 5 years and £18 954 (range 11 664-25 287) over 10 years. Probability of cost per QALY gained below $£ 30000$ was $47 \%$ at 5 years and $57 \%$ at 10 years. Attendance was low: $34 \%$ of intervention families attended no sessions ( $n=48)$; only $47 \%$ completed the course $(n=68)$. Also, $19 \%$ of control families attended a parenting programme before 9-month follow-up.

Conclusions: Our trial has not found evidence of clinical or cost utility for the FLNP in a universal setting. However, low levels of exposure and contamination mean that uncertainty remains.

Trial registration: The trial is registered with Current Controlled Trials ISRCTN13919732.

\section{ARTICLE SUMMARY}

\section{Article focus}

- Evidence that group parenting programmes can change parenting practices and are cost-effective in treating conduct disorder and child behaviour problems is strong. There is also good evidence that they are effective in preventing behavioural problems in high-risk groups identified by socioeconomic deprivation, ethnic group and experience of life events.

- We evaluated the effectiveness and costeffectiveness of the Family Links Nurturing Programme (FLNP) in a randomised controlled trial (RCT) in four deprived areas of South Wales.

- We tested the hypothesis that randomised allocation to the FLNP would be associated with significant advantages over a waiting list control condition, in terms of parenting and child and parental well-being in the short and medium term.

\section{Key messages}

- Our trial has not found evidence of clinical or cost utility for the FLNP in a universal setting.

- However, low levels of exposure and contamination led to reduced power to detect effects; combined with issues relating to the application of RCTs in this setting means that uncertainty remains.

\section{INTRODUCTION}

Parenting is an important determinant of health and well-being. It plays a role in determining future mental health, ${ }^{1-4}$ health-related lifestyles (including healthy eating, ${ }^{5}$ substance misuse, ${ }^{6}$ teenage pregnancy ${ }^{7}$ ), injury rates, ${ }^{8}$ aspects of physical health, ${ }^{3}{ }^{9-12}$ social competence $^{1314}$ and educational achievement. ${ }^{1516}$

Evidence that group parenting programmes can change parenting practices and is cost-effective in treating conduct 


\section{ARTICLE SUMMARY}

Strengths and limitations of this study

- Although recruitment needed to be extended both geographically and temporally, the full sample size was recruited and follow-up rates were exemplary.

- The most important challenges were well-recognised threats to health promotion and disease-prevention trials; those of poor exposure among the intervention group, contamination of the control group. With regard to programme fidelity, programmes run in one of the allocated sites did not meet the standards for running FLNP in all respects.

- A further limitation of our study is the lack of information on the effect of the programme on child quality-adjusted life years.

disorder and child behaviour problems is strong. ${ }^{17}{ }^{18}$ There is also good evidence that they are effective in preventing behavioural problems in high-risk groups identified by socioeconomic deprivation, ethnic group and experience of life events. ${ }^{19-22}$ Although many parenting programmes have been developed and evaluated, ${ }^{23} 24$ most of this evidence is related to two programmes: the Incredible Years Programme 25 and Triple $\mathrm{P}^{27} 28$

Current UK government policies recognise the need for universal parenting support to complement targeted and indicated approaches ${ }^{29-33}$ and the English Department for Education is currently piloting the offer of free vouchers for parenting classes (the CANParent initiative) to all parents in three areas of the country. ${ }^{34}$ Such recommendations derive from observations relating to the prevalence of suboptimal parenting, ${ }^{35}$ the inefficiency of targeting on the basis of identifiable risk factors $^{36} 37$ and the potential for realising change in high risk as well as whole population groups by reducing the stigma which may be attached to targeted parenting support. ${ }^{36-38}$ Given the range and prevalence of health and social outcomes on which parent-child relationships have an influence, ${ }^{2}{ }^{3}$ 5-16 universal approaches are appealing. However, programmes offered universally may suffer from low recruitment and retention rates ${ }^{39}$ and in order to keep costs low programmes may be of low intensity. ${ }^{34}$ Universal programmes can also be challenging to evaluate on several counts. Normal populations may show little change on clinically validated outcome measures, control groups may experience contamination ${ }^{40}$ and, because the effects of parenting on health and social outcomes appear to be life long, costeffectiveness, essential to inform decision making, may be difficult to establish.

With the exception of the Triple $\mathrm{P}^{27}{ }^{28}$ a suite of parenting interventions which can be offered in combination or singly, trials of universal parenting programmes are relatively rare. Two brief group-based parenting programmes have been subject to randomised controlled trials (RCTs); the first a cluster trial in Australia with parents of 8-month-olds ${ }^{41}$ and the second an individually randomised trial in Germany with preschoolers. ${ }^{42}$ In these trials, although there has been some indication of changes in parental attitudes, no changes in parenting or child outcomes have been observed. In contrast, trials of Triple $\mathrm{P}$ have been successful in showing effects on parenting and child behavioural outcomes including child abuse. Various combinations of universal approaches to Triple $\mathrm{P}$ have been subject to large quasi experimental designs at area level ${ }^{43-45}$ and smaller studies with individual randomised designs. ${ }^{46-48}$

The Family Links Nurturing Programme ${ }^{49}$ (FLNP) has much in common with Incredible Years and the groupbased component of Triple $\mathrm{P}$ and, like these programmes, meets the criteria for effective parenting programmes specified in the recently updated National Institute for Health and Care Excellence Guidelines for the prevention and treatment of conduct disorder. ${ }^{50}$ Like Incredible Years and Triple P, FLNP not only covers the principles of positive behaviour management but also addresses the emotional underpinning of problem behaviour and the effect on parenting of the parents' own childhood experiences. It therefore focuses as much on the child's and parent's emotional and social well-being as it does on problem behaviour. The evidence base for FLNP includes qualitative research showing that parents recruited through schools value the programme and perceive it to have an impact on family relationships, children's behaviour and their own mental health ${ }^{51}$; 'before and after' studies in community groups showing impact on self-report measures of relationship quality and well-being ${ }^{52}$; and routine evaluation by parents attending programmes showing that the great majority value the programme. ${ }^{53}$

We evaluated the effectiveness and cost utility (as part of a cost consequences analysis) of the programme in an RCT in four deprived areas of South Wales. We tested the hypothesis that participation in the parenting intervention would be associated with significant advantages over a waiting list control condition, in terms of parenting and child and parental well-being in the short and medium term.

\section{METHODS}

This pragmatic trial was designed in accordance with the consolidated standards of reporting trials guidelines ${ }^{54}$ and was conducted by a research team independent of the programme, with an independent trial steering committee including chairman, parental representation and members with expertise in statistics; early years' intervention studies; and child psychiatry. We obtained informed consent from all participants before randomisation and data collection.

\section{Study population}

This trial took place between December 2008 and January 2011 at four sites (Cardiff, Newport, Torfaen and Caerphilly) in South Wales. Parents with children aged 2-4 years living in the catchment area of 'Flying 
Start' early years centres who had not previously attended an FLNP were eligible for recruitment.

In total, 1323 families were identified as potentially eligible and approached by Flying Start practitioners who offered a participant information leaflet and put interested families in contact with the research team for more information. Recruitment was in five phases tied to the start time of each course: January, April and September 2009 and January and May 2010. Of families approached, $27 \%$ (286) were recruited; two short of the planned sample size of 288. One parent was mistakenly randomised before consent, so the CONSORT flowchart shows a total of 287 families randomised (figure 1). Recruitment rates improved during the trial. ${ }^{55}$ Retention to follow-up of $89 \%$ at 3 months and $84 \%$ at 9 months exceeded pre trial estimations.

\section{Intervention}

FLNP $^{49}$ is a structured, manualised course comprising of an introductory 'coffee morning', followed by 10 weekly $2 \mathrm{~h}$ sessions for groups of 6-10 parents which aims to help parents understand and manage feelings and behaviour, improve relationships at home and in school, improve emotional health and well-being and develop the self-confidence and self-esteem which are essential for effective parenting and learning. It offers support to help parents build on pre-existing parenting skills, use positive behaviour management, communication and relationship strategies and look after their own emotional needs, so they can parent more effectively.

The programme is run by a minimum of two facilitators trained over a 4-day programme with a refresher day 1 year later. As part of the contract provider, organisations agree to offer two supervision sessions to facilitators during the course of each programme. The programme provides experiential learning through the use of guided discussion and role play and a copy of the programme book, the 'Parenting Puzzle'. ${ }^{9}$ Parents try out new skills at home each week and report back on the achievements they made at the next session. The programme was delivered as per usual practice in these localities with no additional training or supervision. It was delivered in term

Figure 1 CONSORT flow chart.

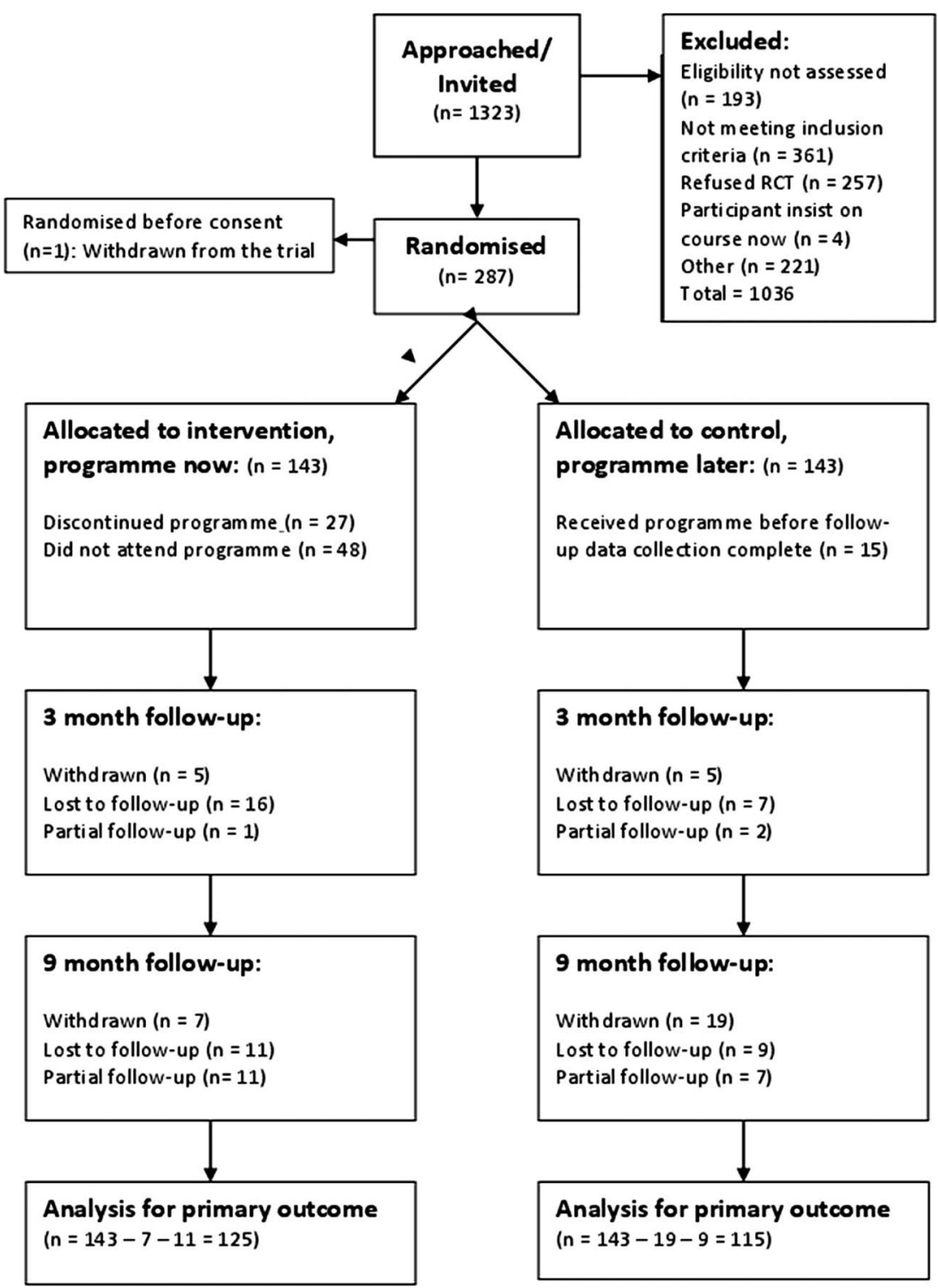


times and entry to the group was closed after the third session In order to deliver viable groups, parents who were not taking part in the trial attended groups with parents who were. Families randomised to the control arm of the trial were offered usual practice, including advice and other forms of support available in the locality during the trial period. Participants agreed at recruitment not to attend the FLNP until after the 9-month follow-up data collection period was complete if randomised to the control arm.

\section{Outcome measures}

All measures were collected during a home visit to the families at baseline (within 2 months of the start of the programme) and at 9 months from baseline (ie, 6 months postcompletion of intervention).$^{55}$ In addition, self-completion questionnaires covering the parent/selfreport outcomes were collected at baseline, 3 months and 9 months. ${ }^{55}$ Data on the resources associated with the implementation of FLNP were collected from structured interviews with key staff at each of the four study sites, collection of financial information at each site (eg, estimates of room hire and crèche facilities) and discussions with the main trial team. ${ }^{55}$

\section{Primary outcome measure}

The primary outcome was a composite index providing two scales representing negative parenting and supportive parenting measured at baseline and 9 months. These scales were the same as the parenting outcomes previously used in a national evaluation of an early years programme in England (Sure Start) ${ }^{56}$ except that we substituted an adapted Mothers' Object Relations Scales (MORS) ${ }^{57}$ for the Pianta Child Parent Relationship Scale. ${ }^{58}$ Our measure was thus derived from observations of maternal and child interactions (tone of voice, responsiveness, affection, spontaneous praise and scolding or hitting) made during the researcher visit and scored dichotomously, questions relating to physical punishment and observation of the orderliness of the home all recorded according to the HOME inventory, ${ }^{59}$ and responses to the MORS adapted by substituting 'child' for 'baby' collected at the same visit. ${ }^{57}$ The latter scale produced two factors (warmth and invasiveness) corresponding closely to the conflict and closeness scales on the Pianta scale used in Sure Start. Factor analysis of the HOME observations produced two scales as in Sure Startresponsivity and acceptance. Overall factor analysis showed very similar findings to those derived in Sure Start. Given the much larger sample in the Sure Start study, variables in this study were weighted with Sure Start weightings:-negative parenting-0.06, $-0.09,0.70,0.60,0.80$ and -0.53 , for responsivity, acceptance, harsh discipline and chaos HOME scores and invasiveness and warmth from the MORS, respectively; supportive parenting $0.80,0.69,0.20,-0.24$, -0.14 and 0.39 , for responsivity, acceptance, harsh discipline and chaos HOME scores and invasiveness and warmth from the MORS, respectively. Both negative and supportive parenting scales were approximately normally distributed.

\section{Secondary outcome measures}

A range of secondary outcome measures was used to capture changes in health and well-being in parents and children. To measure changes in the child's behaviour, we used the preschool version of a clinically validated measure of childhood emotional and behavioural problems the Parent Account of Child Symptoms (PrePACS) ${ }^{60}$ Data on positive and negative interactions were gathered using a video of a child's mealtime coded according to the Mellow Parenting Scheme ${ }^{61}$ and a 5 min speech sample capturing parents' descriptions of their children and their relationship with each child, coded according to the warmth of their initial statement and the percentage of negative comments about the child. ${ }^{62}$ Speech samples and videos were coded following the training of researchers by the developer according to the developer's manuals. ${ }^{61}{ }^{62}$ Researchers received training in the administration of the PrePACS from the developer.

To measure the child's well-being, we used the PedsQL: parent report, ${ }^{63}$ a generic health-related quality-of-life measure. Parental well-being was assessed using the Warwick-Edinburgh Mental Well-being Scale (WEMWBS), ${ }^{64}$ Parenting Stress Index ${ }^{65}$ and the SF-12, ${ }^{66}$ from which SF-6D scores were generated to derive utility values for the estimation of the cost utility of FLNP. ${ }^{67}$ These questionnaires were collected pre intervention, immediately postintervention ( 3 months), and 9 months (6 months postprogramme) ${ }^{55}$

\section{Economic evaluation}

A cost utility analysis (CUA) was undertaken from the perspective of the UK NHS and Social Services. The cost of implementing FLNP was used in conjunction with differences in quality-adjusted life years (QALYs) generated from the SF-6D utilities derived from the SF-12, ${ }^{66}{ }^{67}$ collected from the parents only at baseline and 9 months. The utilities were assumed to remain constant over time, with missing values imputed using predictive mean matching and complete cases only to generate estimates of the cost per QALY gained from FLNP over 5 and 10 -year time horizons, based on linear extrapolation of effect over time. A series of sensitivity analyses was undertaken to assess the impact of changes in cost, removing the assumption of benefits remaining constant over time to a scenario that benefits would gradually reduce to zero over time and using imputed values of effect.

Data were collected on costs of implementing the FLNP in the categories of facilitator and administration costs, training, venue hire, crèche provision, course materials and refreshments. The grade and mix of staff used to deliver FLNP programmes varied within and between each study site and so costs were estimated for each site and the mean cost per programme and per family attending derived from these estimates. Implementation costs associated with the programme were estimated by multiplying resource usage by unit 
costs (where available) sourced from published 2009 PSSRU reference costs for health and social care ${ }^{68}$ or from local costs provided during interviews.

\section{Programme fidelity}

A senior practitioner from Family Links assessed the fidelity of programme delivery using video recordings of three randomly selected sessions for each programme coded against a structured tool which scores sessions from 1 (low fidelity) to 4 (high fidelity) on nine dimensions. One session out of the three selected sessions was watched in its entirety; if this reached a satisfactory standard, the practitioner watched $15-30 \mathrm{~min}$ of the other two sessions to ensure consistency. If the first session was not satisfactory, then the other two sessions were assessed in their entirety.

\section{Sample size}

We aimed to recruit 144 families into each of the control and intervention groups (288 in total). Assuming a drop-out rate from the trial of approximately $20 \%$; this sample size was sufficient to detect a standardised effect size of 0.4 at $85 \%$ power and $\alpha$ of 0.05 in the primary outcome measure if there was no clustering and a standardised effect size of 0.6 allowing for clustering by course with an intraclass correlation coefficient of 0.178 or less; for this sample size calculation, we conservatively imagined clustering within control families as well as intervention families. As the sample size was specified in terms of the number of families rather than the number of children, correlation between siblings in the same family was not considered at the design stage. No interim analyses were planned or conducted.

\section{Randomisation}

We stratified randomisation by site and maintained balance between groups within each stratum using a minimisation method using computer-generated random numbers to give an equal chance of allocation to each group when groups within the stratum were balanced and an increased chance (0.667) of allocation to the smaller group when there was an imbalance within the stratum. ${ }^{69}$ The randomisation service at Warwick Clinical Trials Unit generated the random allocation sequence and informed the FLNP co-ordinators of group allocation following recruitment of a family by the researchers. This ensured the concealment from both families and researchers prior to initial consent being given and from the researchers throughout the trial. $^{55}$

\section{Blinding}

The participants and FLNP coordinators knew the allocation to intervention or waiting list control. The researchers were blind to group allocation, except on rare occasions when families disclosed their assignment status during postcourse interviews. Researchers who did not know the families coded videos and speech samples and all data analyses were carried out blind to group allocation.

\section{Analysis strategy}

The primary analysis for the primary as well as secondary outcome measures was carried out by treatment allocated. Positive change scores indicate a benefit to parenting or well-being. A per protocol analysis compared outcomes for families in which parents did not attend an FLNP session to outcomes for families in which parents attended six or more FLNP sessions. For both treatment allocated and per protocol analysis, we report complete cases analysis results for all outcomes. In all analyses, we fitted multilevel models with a random effects term for course and for outcomes corresponding to individual child data and a random effects term for family. For the families in the control, the betweenfamily variance was assumed to be equal to that between families in the intervention group attending different courses; terms in the model were thus included as if each control family formed their own course, so that they contributed to estimating the between course random effect variance. For sensitivity analysis, we performed multiple imputation using predictive mean matching ${ }^{70}$ and results are reported for the primary

\begin{tabular}{lcc}
\multicolumn{4}{l}{ Table 1 Baseline characteristics of families } \\
\hline Characteristic & Control & FLNP \\
\hline Number of parents & 143 & 143 \\
Number of children & 145 & 150 \\
Negative parenting: mean & $11.07(10.20)$ & $10.61(11.36)$ \\
(SD) & & \\
Supportive parenting: & $26.00(2.64)$ & $25.88(2.92)$ \\
mean (SD) & & \\
Parent gender: $\mathrm{n}(\%)$ of & $139(99.3)$ & $136(95.1)$ \\
females & & \\
Number of parents with & $49(34.3)$ & $46(32.4)$ \\
partner: $\mathrm{n}(\%)$ & & \\
Parent working: $\mathrm{n}(\%)$ & $112(78.3)$ & $112(78.9)$ \\
working & & \\
Parent's ethnicity: $\mathrm{n}(\%)$ & $103(72.0)$ & $100(69.9)$ \\
White British & $21(14.7)$ & $22(15.4)$ \\
White other & $4(2.8)$ & 0 \\
Black African & 0 & $1(0.7)$ \\
Black Caribbean & $9(6.3)$ & $6(4.2)$ \\
Asian Indian/Pakistani/ & & \\
Bangladeshi & 0 & $2(1.4)$ \\
Asian Chinese & $6(4.2)$ & $12(8.4)$ \\
Other & & \\
Parent's education: $\mathrm{n}(\%)$ & $26(18.4)$ & $28(20.1)$ \\
NVQ & $46(32.6)$ & $39(28.1)$ \\
GCSE/O levels & $16(11.3)$ & $15(10.8)$ \\
A levels & $17(12.1)$ & $15(10.8)$ \\
Degree & $4(2.8)$ & $3(2.2)$ \\
Higher degree & $24(17.0)$ & $24(17.3)$ \\
None & $8(5.7)$ & $15(10.8)$ \\
Other & & \\
\hline FLNP, Family Links Nurturing Programme. & \\
& &
\end{tabular}




\begin{tabular}{|c|c|c|c|c|c|c|c|c|}
\hline \multirow[b]{2}{*}{ Outcomes } & \multicolumn{3}{|c|}{ Control } & \multicolumn{3}{|c|}{ Intervention } & \multirow[b]{2}{*}{$\begin{array}{l}\text { Effect size }(95 \% \mathrm{Cl}) \text {, } \\
\text { p value† analysis by } \\
\text { treatment allocated }\end{array}$} & \multirow[b]{2}{*}{$\begin{array}{l}\text { Effect size }(95 \% \mathrm{Cl}), \\
\mathrm{p} \text { value } † \neq \text { per protocol } \\
\text { analysis }\end{array}$} \\
\hline & $\mathrm{n}^{*}$ & $\begin{array}{l}\text { Baseline score } \\
\text { (SD) }\end{array}$ & $\begin{array}{l}\text { Change at } \\
9 \text { months (SE) }\end{array}$ & $\mathrm{n}^{*}$ & $\begin{array}{l}\text { Baseline score } \\
\text { (SD) }\end{array}$ & $\begin{array}{l}\text { Change at } \\
9 \text { months (SE) }\end{array}$ & & \\
\hline \multicolumn{9}{|l|}{ Primary outcomes } \\
\hline Negative parenting & 106 & $11.04(10.27)$ & $1.41(0.96)$ & 115 & $10.72(11.62)$ & $2.31(1.02)$ & $0.90(-1.90$ to 3.69$), 0.52$ & $1.52(-1.58$ to 4.61$), 0.34$ \\
\hline $\begin{array}{l}\text { Supportive } \\
\text { parenting }\end{array}$ & 106 & $26.04(2.75)$ & $0.38(0.27)$ & 115 & $25.82(3.00)$ & $0.55(0.28)$ & $0.17(-0.61$ to 0.94$), 0.67$ & $0.43(-0.44$ to 1.30$), 0.33$ \\
\hline \multicolumn{9}{|l|}{ Secondary outcomes } \\
\hline SF-12 & 110 & $0.67(0.09)$ & $0.01(0.01)$ & 121 & $0.68(0.08)$ & $0.02(0.07)$ & $0.01(-0.01$ to 0.02$), 0.60$ & $0.01(-0.02$ to 0.03$), 0.69$ \\
\hline $\begin{array}{l}\text { Parenting Stress } \\
\text { Index }\end{array}$ & 99 & $123.33(21.49)$ & $5.88(1.78)$ & 106 & $124.13(22.39)$ & $5.08(1.72)$ & $-0.80(-5.74$ to 4.14$), 0.75$ & $2.88(-2.78$ to 8.54$), 0.32$ \\
\hline PedQL & 112 & $58.60(8.90)$ & $-0.20(0.81)$ & 118 & $58.51(7.87)$ & $1.30(0.80)$ & $1.50(-0.74$ to 3.74$), 0.19$ & $1.10(-1.37$ to 3.56$), 0.38$ \\
\hline WEMWBS score & 113 & $40.61(9.01)$ & $2.53(0.81)$ & 121 & 39.54 (9.71) & $3.03(0.83)$ & $0.49(-1.82$ to 2.80$), 0.67$ & $2.02(-0.56$ to 4.59$), 0.13$ \\
\hline \multicolumn{9}{|l|}{ PrePACS } \\
\hline Hscale (weekly) & 114 & $11.70(6.68)$ & $2.57(0.66)$ & 129 & $12.14(6.84)$ & $3.43(0.63)$ & $0.86(-0.95$ to 2.66$), 0.35$ & $1.61(-0.46$ to 3.67$), 0.13$ \\
\hline Hscale (typical) & 115 & $13.78(7.02)$ & $2.62(0.69)$ & 129 & $13.41(7.49)$ & $2.58(0.82)$ & $-0.03(-2.18$ to 2.12$), 0.98$ & $-0.06(-2.29$ to 2.18$), 0.96$ \\
\hline Cscale (weekly) & 116 & $16.09(8.38)$ & $1.23(0.83)$ & 128 & $16.07(9.45)$ & $1.27(1.01)$ & 0.03 ( -2.57 to 2.64$), 0.98$ & $1.94(-0.77$ to 4.65$), 0.16$ \\
\hline Cscale (typical) & 116 & $16.58(8.39)$ & $1.50(0.79)$ & 127 & $16.07(9.94)$ & $1.38(0.88)$ & $-0.12(-2.47$ to 2.24$), 0.92$ & $1.29(-1.27$ to 3.84$), 0.32$ \\
\hline Internalising (Freq) & 110 & $5.66(3.43)$ & $0.26(0.33)$ & 122 & 5.44 (3.19) & $0.40(0.34)$ & $0.13(-0.82$ to 1.09$), 0.78$ & $-0.08(-1.16$ to 1.00$), 0.89$ \\
\hline $\begin{array}{l}\text { Internalising } \\
\text { (reassure) }\end{array}$ & 110 & $5.46(3.73)$ & $0.39(0.42)$ & 126 & $5.18(3.68)$ & $0.10(0.40)$ & $-0.29(-1.43$ to 0.85$), 0.62$ & $-1.02(-2.34$ to 0.30$), 0.13$ \\
\hline $\begin{array}{l}5 \text { min speech } \\
\text { sample (warmth of } \\
\text { opening } \\
\text { statements) }\end{array}$ & 107 & $6.30(1.38)$ & $0.29(0.14)$ & 124 & $6.48(1.30)$ & $0.30(0.14)$ & $0.01(-0.39$ to 0.40$), 0.97$ & $0.14(-0.30$ to 0.58$), 0.54$ \\
\hline $\begin{array}{l}5 \text { min speech } \\
\text { sample } \\
\text { (\% negative } \\
\text { comments) }\end{array}$ & 105 & $0.43(0.26)$ & $0.07(0.03)$ & 117 & $0.41(0.27)$ & $0.05(0.03)$ & $-0.01(-0.10$ to 0.07$), 0.75$ & $0.06(-0.03$ to 0.15$), 0.21$ \\
\hline \multicolumn{9}{|l|}{ Meal-time video } \\
\hline $\begin{array}{l}\text { Proportion of } \\
\text { negative } \\
\text { interactions }\end{array}$ & 84 & $0.12(0.16)$ & $-0.02(0.02)$ & 87 & $0.14(0.15)$ & $0.03(0.02)$ & 0.05 (-0.01to 0.11$), 0.13$ & $0.03(-0.04$ to 0.10$), 0.41$ \\
\hline $\begin{array}{l}\text { Rate of negative } \\
\text { interactions } \\
\text { (length) }\end{array}$ & 84 & $0.49(0.73)$ & $-0.02(0.10)$ & 87 & $0.52(0.72)$ & $0.09(0.10)$ & $0.11(-0.19$ to 0.40$), 0.46$ & 0.09 ( -0.23 to 0.41$), 0.59$ \\
\hline $\begin{array}{l}\text { Rate of positive } \\
\text { interactions } \\
\text { (length) }\end{array}$ & 84 & $3.65(2.57)$ & $-0.14(0.46)$ & 87 & $3.13(2.24)$ & $0.62(0.46)$ & $0.75(-0.53$ to 2.04$), 0.25$ & $0.56(-0.87$ to 1.99$), 0.44$ \\
\hline $\begin{array}{l}\text { Rate of negative } \\
\text { interactions } \\
\text { (involvement) }\end{array}$ & 72 & $0.68(0.96)$ & $-0.19(0.19)$ & 73 & $0.72(0.86)$ & $-0.16(0.19)$ & $0.03(-0.50$ to 0.57$), 0.91$ & $0.07(-0.52$ to 0.66$), 0.82$ \\
\hline
\end{tabular}




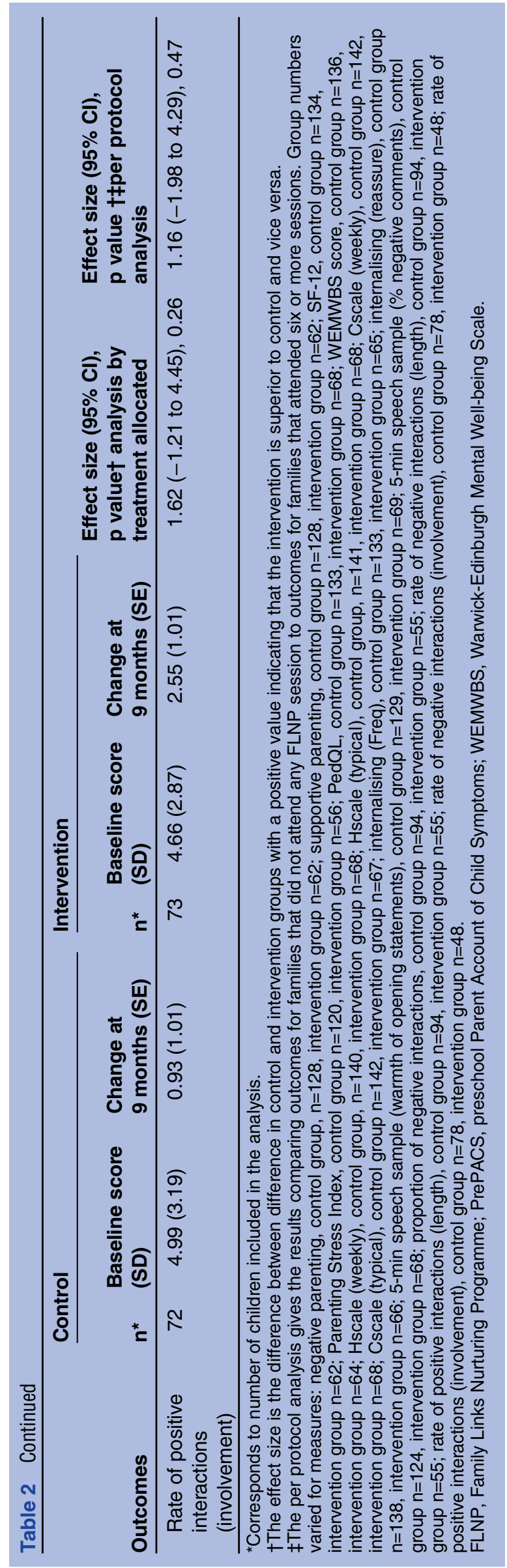

outcomes and SF-12. For each outcome, we created 10 imputed datasets using the Hmisc package in the $\mathrm{R}$ statistical programme and combined the analysis results from the 10 imputed datasets using Rubin rules. ${ }^{70}$

\section{RESULTS}

The groups had similar baseline characteristics in terms of gender, employment, ethnicity, parents' education and parenting scores (table 1).

\section{Uptake of intervention}

Forty-eight families (34\%) randomly allocated to the intervention (FLNP) group did not attend any sessions, a further $27(19 \%)$ discontinued the programme in the first three sessions. Fifteen control group families (10\%) attended an FLNP before 9-month follow-up and a further $13(9 \%)$ control group parents attended other parenting support programmes during the trial.

\section{Main findings: effectiveness}

Although both groups improved over time, and the intervention group more so than the control group, the difference in change between groups was not statistically significant at 9 months (change in negative parenting: control group $+1.41,95 \% \mathrm{CI}-0.47$ to 3.30 ; FLNP group $+2.31,95 \%$ CI 0.21 to 4.41 ; difference in change +0.90 , $95 \%$ CI -1.90 to 3.69 ; change in supportive parenting: control group $+0.38,95 \%$ CI -0.15 to 0.91 ; FLNP group $+0.55,95 \%$ CI -025 . to 1.12; difference in change, +0.17 , 95\% CI-0.61 to 0.94). This pattern of results was repeated in 12 of the 17 secondary outcomes: SF-12; PedsQL; WEMWBS; PrePACS hyperactivity, conduct and internalising (frequency); speech sample warmth of opening statement and video proportion of negative interactions, rate of negative interactions (length), rate of positive interactions (length), rate of negative interactions (involvement) and rate of positive interactions (involvement; table 2). The results for the primary outcome measures and SF-12 by treatment as allocated after multiple imputation were also not statistically significant (table 3). At 3 months, the results were similar with no statistically significant differences in change, although observed outcomes favoured the intervention group for three of the four secondary outcomes (table 4). Per protocol analyses reflected these results at both time points (tables 2 and 4 ).

\section{Programme fidelity}

Overall fidelity score for courses in site A was $87 \%$, site B $82 \%$, site C $72 \%$ and site D $60 \%$ (table 5 ). Fidelity in phase 1 courses was $85 \%$, phase $283 \%$, phase $375 \%$, phase $477 \%$ and phase $547 \%$. Fidelity was much lower in phase 5 due in part to the higher proportion of families from site $\mathrm{D}$ in this phase of the trial. The per protocol analysis was repeated comparing the primary outcome measures for control families with families attending six or more FLNP sessions on a course with 
Table 3 Outcomes at 9 months: by treatment as allocated after multiple imputation

\begin{tabular}{lllr}
\hline Outcomes & $\mathbf{n}$ (control) & $\mathbf{n}$ (intervention) & Effect size (95\% Cl), $\mathbf{p ~ V a l u e ~}$ \\
\hline Negative parenting & 145 & 150 & $1.10(-0.66$ to 2.86$), 0.22$ \\
Supportive parenting & 145 & 150 & $0.35(-0.14$ to 0.83$), 0.16$ \\
SF-12 & 143 & 143 & $<0.01(-0.02$ to 0.02$), 0.81$ \\
\hline
\end{tabular}

fidelity of $80 \%$ or more. The estimated differences and between those who attended at least six FLNP sessions with high fidelity $(n=41)$ and control groups $(n=128)$ on supportive and negative parenting and $95 \%$ CI were 0.45 ( -0.50 to 1.41$)$ and 0.00 (-3.45 to 3.45$)$, respectively. Neither difference was statistically significant.

\section{Economic evaluation results}

Costs of the delivery of FLNP varied between sites (table 6) due to difference in staff delivering the programme-the implications of which are explored in the sensitivity analysis. The cost per family per programme (£648) was used as the cost differential between FLNP and control group for the baseline findings. Cost per QALY for FLNP, based on the complete case analysis, was estimated at $£ 34913$ over 5 years and $£ 18954$ over 10 years duration, with the probability that FLNP can be regarded as cost-effective as $36 \%$ against the $£ 20000$ threshold over 5 years, and $51 \%$ over 10 ; and $47 \%$ against the $£ 30000$ threshold over 5 years, and $57 \%$ over 10 (table 7 ).

Sensitivity analyses show that the impact of cost is highly relevant in determining the likelihood that FLNP can be viewed as representing value for money. For example, the distribution of implementation costs across a larger number of attendees (cost per family of $£ 399$ when 13 parents attending) increases the likelihood that the programme would be cost-effective given a $£ 20000$ threshold from $36 \%$ to $49 \%$ - using a 5-year time horizon; whereas a smaller number of attendees (cost per parent of $£ 865$ when six parents attending) reduce the likelihood to $26 \%$ with a 5 -year time horizon.

Relaxing the assumption that benefits remain constant over time and assuming that the effect diminishes to zero by the end of the time period considered results in an estimated cost per QALY of $£ 56885$ for the 5-year duration (probability cost-effective at $£ 20000=30 \%$ ) and $£ 29664$ for the 10 -year time horizon (probability at the $£ 20000$ threshold $=44 \%$; table 8 ).

\section{DISCUSSION}

This trial investigated the effectiveness of an existing parenting programme offered on a universal basis in deprived areas in the context of an open access early years programme offering a wide range of support for parenting. Although recruitment needed to be extended both geographically and temporally, ${ }^{55}$ the full sample size was recruited and follow-up rates were exemplary.
The results of the treatment allocated analyses showed no statistically significant difference between intervention and control groups on any of the outcome measures. For most outcomes, improvement was observed in both groups with families in the FLNP arm of the trial improving more than those in the control arm by a small and not statistically significant amount, the differences observed being very much smaller than those which the trial was powered to detect.

The 3-month results suggest that differences in parental well-being were greatest at the end of the programme and declined over the next 6 months. In contrast, the observed differences in child well-being as measured by the PedsQL increased over time.

Our economic analyses do not provide evidence that FLNP represents value for money, but are based on an estimate of parental utility alone. A recent study of the cost-effectiveness of the Incredible Years Programme in a targeted population on key outcomes in later life has demonstrated favourable long-run economic returns. ${ }^{71}$

\section{Limitations of the study}

The most important challenges were well-recognised threats to health promotion and disease-prevention trials; those of poor exposure among the intervention group, contamination of the control group and varying programme fidelity. Our predicted programme nonattendance and dropout rate in the first three sessions, based on the provision of this programme in other settings, ${ }^{52}$ was $16 \%$. In the event, the rate was $53 \%$. It is possible that the trial setting may have influenced attendance rates by preferentially recruiting families who were not ready to change ${ }^{40}$ as such families were more likely to be willing to be randomised. Testing the effectiveness of an existing programme in a pragmatic trial makes it challenging to restrict access to the programmes in control group families and in this trial $10 \%$ of the control group violated the rules of the trial and attended an FLNP course before the 9-month follow-up. A further $9 \%$ of control parents accessed different parenting support courses available in the locality, but only one of the intervention group families accessed any other type of parenting support. The main trial results therefore compare the changes in parenting and family well-being among a group in which just under one in two parents were exposed to the FLNP with changes among a control group in which roughly one in five parents attended the FLNP or other parenting support programme. Had these factors been entirely responsible 


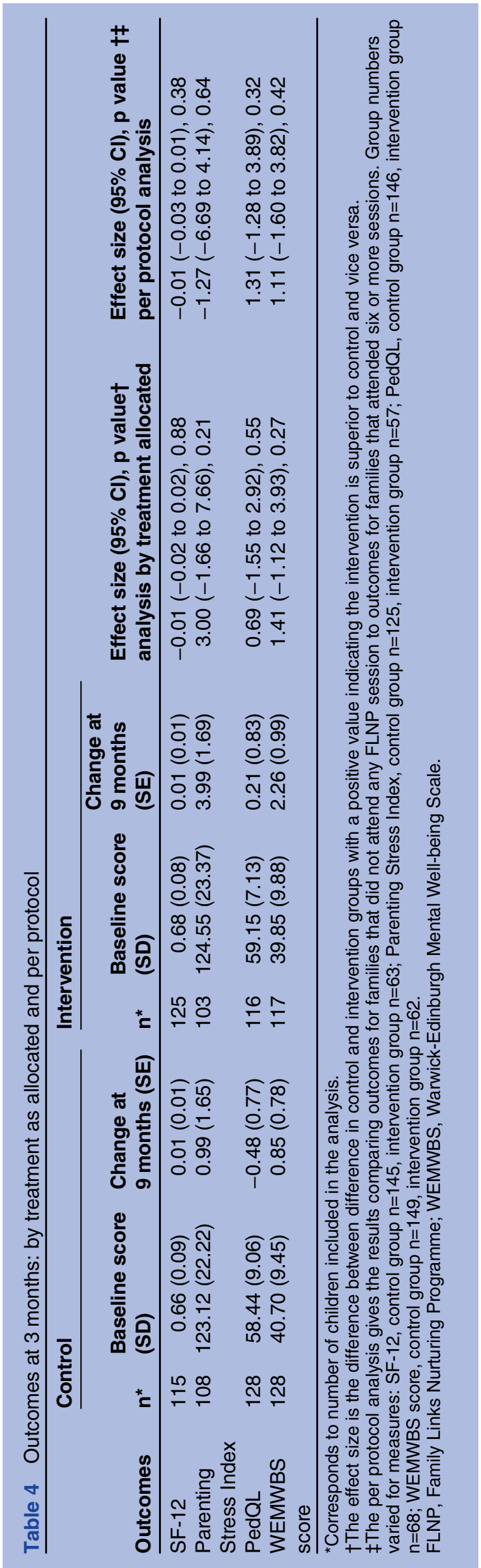

\begin{tabular}{lllll} 
Table 5 Overall fidelity score distributions \\
\hline & \multicolumn{3}{l}{$\begin{array}{l}\text { Percentage of } \\
\text { programme scoring }\end{array}$} \\
\cline { 2 - 5 } Fidelity ranking $\mathbf{1}$ low, $\mathbf{4}$ high) & $\mathbf{1}$ & $\mathbf{2}$ & $\mathbf{3}$ & $\mathbf{4}$ \\
\hline Welcome & 2 & 14 & 38 & 46 \\
Family feedback & 4 & 17 & 44 & 35 \\
Topics before break & 6 & 21 & 40 & 33 \\
Topics after break & 5 & 26 & 43 & 26 \\
Time to have a go & 0 & 22 & 22 & 56 \\
Modelling group rules & 4 & 25 & 38 & 33 \\
Active listening & 0 & 40 & 43 & 17 \\
Empathy & 4 & 25 & 33 & 38 \\
Nurturing environment & 4 & 18 & 31 & 47 \\
\hline
\end{tabular}

for the trial findings, however, we would have expected to see significant results in the per protocol analysis. Changes in the latter analysis were larger than those in the as-allocated analysis, but still did not reach statistical significance.

Our assessment of programme fidelity was constrained by costs. We showed that programmes run in one of the allocated sites did not meet the standards for running FLNP in all respects. It is important to note that the sites running programmes with the highest level of fidelity were those with the highest costs and those with the lowest fidelity, the cheapest to run. We attempted an analysis of results by programme fidelity and also by area, and no statistically significant differences between the groups were found. It may be that our estimates of fidelity were inadequate being based on a random sampling of only one session in most of the programmes.

A further limitation of our study is the lack of information on the effect of the programme on child QALYs. FLNP aims, by improving parenting, to improve children's well-being as well as parent's well-being. In our CUA, we were able only to include change in parental QALYs and the measure used (SF-6D) did not prove sensitive to variation in parental well-being as measured by other instruments. Given that non-significant positive changes in PedsQL scores were considerably greater $(>1$ $\mathrm{SE})$ than the non-significant changes in parental SF-6D scores, including child QALYS could greatly increase cost utility estimates. If it becomes possible in future to estimate QALYs from PedsQL and adopt sensitive measures of parental well-being, it will be possible to revisit these estimates. If, as has been suggested, parenting has a life-course impact on health and social outcomes the relatively short-term outcomes possible in RCTs are inadequate to assess the full benefits and cost savings.

Despite inclusion in data collection, little self-reported information was provided by parents (and verified by contact with the research team) at follow-up on the use of health, social service, educational psychology or criminal justice service contacts during the course of the trial, attributable to these events or to involvement in the FLNP, with insufficient 
Table 6 Summary of costs associated with implementation

\begin{tabular}{|c|c|c|c|c|c|}
\hline Total costs & Site A & Site B & Site C & Site D & Total \\
\hline Salaries* & $£ 63002$ & $£ 33341.4$ & $£ 52772$ & $£ 44423$ & $£ 193538$ \\
\hline Training & $£ 5801.90$ & $£ 4733.90$ & $£ 5081.90$ & $£ 5144$ & $£ 20761.70$ \\
\hline Room hireł & $£ 3883.89$ & $£ 3003$ & $£ 3000$ & $£ 3000$ & $£ 12886.89$ \\
\hline Course materials $\ddagger$ & $£ 3750$ & $£ 1625$ & $£ 2250$ & $£ 2250$ & $£ 9875$ \\
\hline Crècheł & $£ 17862.66$ & $£ 17097.96$ & $£ 15000$ & $£ 15000$ & $£ 64960.62$ \\
\hline Total cost & $£ 94300.45$ & $£ 59801.26$ & $£ 78103.90$ & $£ 69827$ & $£ 302022.21$ \\
\hline No of courses & 15 & 13 & 15 & 15 & 58 \\
\hline Cost per 11-week programme & $£ 6286.70$ & $£ 4600$ & $£ 5206.93$ & $£ 4654.47$ & $£ 5207.28$ \\
\hline \multicolumn{5}{|c|}{ Mean cost per 11-week programme } & $£ 5187.03(\mathrm{SD}=782.71)$ \\
\hline \multicolumn{5}{|c|}{ Mean cost per family per programme (based on 8 parents attending) } & $£ 648.38(\mathrm{SD}=97.84)$ \\
\hline \multicolumn{5}{|c|}{ Cost per family per programme (based on 13 parents invited to attend) } & 399 \\
\hline \multicolumn{5}{|c|}{ Cost per family per programme (based on 6 parents completing the programme) } & 865 \\
\hline \multicolumn{6}{|c|}{$\begin{array}{l}\text { *We applied hourly rates based on grade of staff (including on-costs) from PSSRU } 52 ; 34 / \mathrm{h} \text { for health visitor; } 23 / \mathrm{h} \text { for parenting coordinator and } \\
18 / \mathrm{h} \text { for community support worker. Administrative overheads were estimated at } 10 \% \text { direct salary costs in line with recommendations from the } \\
\text { local finance teams. } \\
\text { †Training was based on cost of facilitator ( } 34 / \mathrm{h} \text { for health visitor) and hourly rate for staff to attend initial training from PSSRU }{ }^{68} \text { and top up } \\
\text { sessions including travel costs based on HM revenue and customs approved mileage rates }{ }^{73} \\
\neq \text { Costs were derived directly from financial records and interviews with staff at local sites. }\end{array}$} \\
\hline
\end{tabular}

data to undertake any meaningful analysis. Several of these services are, however, unlikely to be relevant to children of $2-4$ years. This has limited the ability of the study to consider the full range of perspectives in the analysis.

\section{Meanings and implications of the study}

Although outcome measures improved more in the FLNP group than in the control group, the results of this trial fail to show that FLNP improved parenting or child or parental well-being more than could be expected by chance and do not provide evidence that FLNP represents value for money. Three main reasons for these negative results warrant consideration. The first is that the FLNP may not be effective in changing parenting in a universal setting. It would appear that programmes like Triple $\mathrm{P}$ which focus primarily on behaviour management are effective in a universal setting and this may simply be a better approach. Triple $\mathrm{P}$ is a more costly programme than FLNP. It can be offered more intensively with five different levels of intervention for different levels of need, including universal media coverage, and well-resourced trials have been able to offer the programme in this way. FLNP is a stand-alone group-based programme which may work synergistically with other parenting interventions including media coverage. In this trial, both intervention and control group parents were exposed to general parenting interventions available in the Flying Start setting.

FLNP aims to address the promotion of mental wellbeing in parents and children as well as behaviour management, thus potentially influencing resilience to a variety of mental health problems including anxiety and depression throughout the life course. In this way, it does more to address the aims of the current government strategy No Health without Mental Health ${ }^{32}$ than the better researched behaviour management programmes. However, as we failed to identify any change in parenting or family well-being as a result of the trial, it may be that the contents and approach of the programme need revisiting.

The second reason why the trial may have produced negative results is that the programme was implemented

Table 7 Cost utility analysis

\begin{tabular}{llll}
\hline & FLNP & Control & Difference \\
\hline Cost per programme (£) 8 participants & 648 & 0 & 648 \\
Cost per programme (£) 6 participants & 895 & 0 & 895 \\
Cost per programme (£) 13 participants & 399 & 0 & 399 \\
Complete cases & & & 0.019 \\
QALY gain (5 years) & 0.053 & 0.035 & 0.034 \\
QALY gain (10 years) & 0.098 & 0.064 & Probability $\leq £ 20000=36 \%$ \\
Cost per QALY gained (5 years) (£) & 34913 & & Probability $\leq £ 30000=47 \%$ \\
& & & Probability $\leq £ 20000=51 \%$ \\
Cost per QALY gained (10 years) (£) & 18954 & & Probability $\leq £ 30000=57 \%$ \\
& & &
\end{tabular}


Table 8 Sensitivity analysis

\begin{tabular}{|c|c|c|c|c|}
\hline & $\begin{array}{l}\text { Cost per QALY } \\
(5 \text { years }(£)\end{array}$ & $\begin{array}{l}\text { Probability cost } \\
\text { effective }\end{array}$ & $\begin{array}{l}\text { Cost per QALY } \\
\text { (10 years }(£)\end{array}$ & $\begin{array}{l}\text { Probability cost } \\
\text { effective }\end{array}$ \\
\hline Baseline & 36000 & $\begin{array}{l}35 \% @ £ 20000 \\
46 \% @ £ 30000\end{array}$ & 19115 & $\begin{array}{l}51 \% @ £ 20000 \\
58 \% @ £ 30000\end{array}$ \\
\hline Lowest cost & 21485 & $\begin{array}{l}49 \% @ £ 20000 \\
55 \% @ £ 30000\end{array}$ & 11664 & $\begin{array}{l}58 \% @ £ 20000 \\
62 \% @ £ 30000\end{array}$ \\
\hline Highest cost & 46578 & $\begin{array}{l}26 \% @ £ 20000 \\
40 \% @ £ 30000\end{array}$ & 25287 & $\begin{array}{l}45 \% @ £ 20000 \\
53 \% @ £ 30000\end{array}$ \\
\hline $\begin{array}{l}\text { Benefits diminish to zero } \\
\text { over time }\end{array}$ & 56885 & $\begin{array}{l}30 \% @ £ 20000 \\
40 \% @ £ 30000\end{array}$ & 29664 & $\begin{array}{l}44 \% @ £ 20000 \\
50 \% @ £ 30000\end{array}$ \\
\hline Imputed values & 34122 & $\begin{array}{l}37 \% @ £ 20000 \\
47 \% @ £ 30000\end{array}$ & 18525 & $\begin{array}{l}51 \% @ £ 20000 \\
58 \% @ £ 30000\end{array}$ \\
\hline
\end{tabular}

poorly in this setting. Even Triple $\mathrm{P}$ has shown negative results in independent pragmatic trials ${ }^{72}$ due in all likelihood to inadequate implementation. This trial measured changes due to the FLNP in an established setting where no attempt was made to improve implementation for the trial. It may be that the training and supervision of these facilitators were not sufficient to maintain highquality provision. Our assessment of fidelity was constrained by resources. Although it was judged as adequate by the provider in three of the four settings, we did not examine every session and would not have known if problems had arisen in other sessions. We were also unable to assess the quality of supervision offered to facilitators by the provider organisations.

Finally, the trial may have produced negative results because of the research design.

It is possible that the outcome measures we selected were not sensitive to changes which did occur. The observations in the HOME inventory, the 5 min speech sample and the meal-time video were made over a relatively short visit and some parents refused the video. ${ }^{55}$ Although objective measures are highly valued, the parenting recorded is that of parents on their best behaviour. Against this, we did not identify changes in parent or child well-being either.

The trial was powered to capture effect sizes reported for parenting trials in general most of which are in clinical or targeted settings where opportunities for improvement are greater than in universal populations. It may be that the trial was underpowered to pick up the level of changes that could represent a valuable change at the population level where smaller differences have great impact because of the numbers involved.

The open access early years centre setting certainly presented challenges including the contamination of the control group due to the ready availability of parenting support, and unusually low levels of attendance at the programme. We have also identified in a separate study ${ }^{40}$ the possibility of a threat to the external validity of the trial. Parents who consented to randomisation may have been different from those who would normally sign up for the FLNP in that some parents did not recognise a need for the programme for themselves and their families. They may therefore have been less ready to change and indeed had less need to change than those who attend the FLNP in the absence of a trial. Together, these factors undoubtedly reduced the study's power to detect any impact of the FLNP.

\section{Future research}

Overall, we have concluded that uncertainty remains about the effectiveness and cost utility of the FLNP in universal settings and that further investigation is required. It may be that, given the multiple challenges faced by trials of this nature, studies need to have more statistical power, be based in settings where contamination can be controlled, employ outcomes that are known to change during the course of the programme and where very high-quality programme implementation can be ensured. In the meanwhile, the results of this trial provide estimates to inform the design of a future trial.

Acknowledgements We are indebted to all the families who took part in this research project and to all the Sure Start/Flying Start staff including Health Visitors who facilitated recruitment by discussing this project with eligible families. We would like to thank all the Family Links Nurturing programme facilitators who ran the programmes on which the trial was based and the managers within each of the four districts; Bernice Bird in Cardiff, Lorraine Childs in Torfaen, Mandy Shide in Newport and Sarah Mutch in Caerphilly for their unstinting support. In addition, the parenting coordinators across the boroughs provided invaluable assistance to the research staff. Marie Thomas and Alexandra Nicholson were valued members of the research team in the early part of the study and we are very grateful for Marie's leadership of the research team and their contributions in many ways to the project. Michael Nugent has supported this project in a number of ways: acting as an advisor to the commissioners and then organising the independent trial steering group and facilitating and its meetings. We are grateful to him and to the members of the independent trial steering committee who gave their time to ensure that the trial was run to the highest possible standards. Finally, this project would not have been possible without the financial support of the Welsh Assembly Government and the four local authorities in south Wales. We would like to express our gratitude to Avril Hooper in particular who has led the commissioning of this research from conception to completion.

Contributors DS, HAS, NS and SSB conceived the idea of the study and were responsible for the design of the study. PKK, NS, BS, DF and CJP were responsible for undertaking the data analysis and producing the tables and figure. RA, SW and LW were repsonsible for collecting the data and provided 
input into the data analysis. The initial draft of the manuscript was prepared by DS, HAS and SSB and then circulated among all the authors for critical review.

Funding This trial was funded by a consortium of four local authorities in South Wales: Cardiff, Torfaen, Newport and Caerphilly and the Welsh Assembly Government and amounted to $£ 530486$. The funders convened a commissioner group and were represented on the Trial Steering Group, but had no involvement in the management of the trial. Additional funding to analyse the video outcome data was provided by the Esme Fairburn Foundation and the Waterloo Foundation.

\section{Competing interests None.}

Ethics approval Ethical approval for the overall study was granted by the North West Wales Research Ethics Committee $(08 /$ Wno01/50) on 6 October 2008. Minor adaptations to the outcome measures, indicated following piloting, were subsequently approved (AM01) on 22 December 2008.

Provenance and peer review Not commissioned; externally peer reviewed.

Data sharing statement No additional data are available.

Open Access This is an Open Access article distributed in accordance with the Creative Commons Attribution Non Commercial (CC BY-NC 3.0) license, which permits others to distribute, remix, adapt, build upon this work noncommercially, and license their derivative works on different terms, provided the original work is properly cited and the use is non-commercial. See: http:// creativecommons.org/licenses/by-nc/3.0/

\section{REFERENCES}

1. Department for Education and Skills: Curriculum Guidance for the Foundation Stage [cited 26.9.12]. http://www.standards.dfes.gov.uk/ primary/publications/foundation_stage/63593

2. Carlson E. A prospective longitudinal study of attachment disorganisation/disorientation. Child Dev 1998;69:1107-28.

3. Weich SS, Patterson J, Shaw R, et al. Family relationships in childhood and later psychopathology: a systematic review of longitudinal studies. Br J Psychiatry 2009;194:392-8.

4. Repetti RL, Taylor SE, Seeman TE. Risky families: family social determinants and the mental and physical health of offspring Psychol Bull 2002;128:330-66.

5. Kremers SPJ, Brug J, De Vries $\mathrm{H}$, et al. Parenting style and adolescent fruit consumption. Appetite 2003;41:43-50.

6. Cohen D, Richardson J, Labree L. Parenting behaviours and the onset of smoking and alcohol use: a longitudinal study. Pediatrics 1994;94:368-75.

7. Scaramella LV, Conger RD, Simons RL, et al. Predicting risk for pregnancy by late adolescence: a social contextual perspective. Dev Psychol 1998;34:1233-45.

8. Bijur PE, Kurzon M, Hamelsy V, et al. Parent-adolescent conflict and adolescent injuries. J Dev Behav Pediatr 1991;12:92-7.

9. Stewart-Brown S, Fletcher L, Wadsworth MEJ. Parent-child relationships and health problems in adulthood in three UK national birth cohort studies. Eur J Public Health 2005;15:640-6.

10. Belsky J, Bell B, Bradley $\mathrm{RH}$, et al. Socioeconomic risk, parenting during the preschool years and child health age 6 years. Eur $J$ Public Health 2007;17:508-13.

11. Clover RD, Abell T, Becker LA, et al. Family functioning and stress as predictors of influenza B infection. J Fam Prac 1989;28:535-53.

12. Flinn MV, England BG. Social economics and childhood glucocorticoid stress response and health. Am J Phys Anthropol 1997;102:33-53.

13. Sroufe LA, Egeland B, Carlson EA. One social world: the integrated development of parent-child and peer relationships. In: Collins WA, Laursen B, eds. Relationships as Developmental Contexts. Mahwah: Lawrence Erlbaum Associates, 1999:241-61.

14. MacCoby EE, Martin JA. Socialization in the context of the family: parent-child interaction. In: Heatherington EM. ed. Mussen manual of child psychology. New York: Wiley, 1983:1-102.

15. Early Child Care Research Networks. Child care and child development: results of the NICHD study of early child care and youth development. New York: Guildford Press, 2005.

16. Steinberg L, Lamborn SD, Dornbursch SM, et al. Impact of parenting practices on adolescent achievement: authoritative parenting, school involvement, and encouragement to succeed. Child Dev 1992;63:1266-81.
17. Dretzke J, Frew E, Davenport C, et al. The effectiveness and costeffectiveness of parent training/education programmes for the treatment of conduct disorder, including oppositional defiant disorder, in children. Health Technol Assess (Winch Eng) 2005;9:50.

18. Lundahl B, Risser HJ, Lovejoy MC. A meta-analysis of parent training: moderators and follow-up effects. Clin Psychol Rev 2006;26:86-104.

19. Webster Stratton C, Reid JM, Hammond M. Preventing conduct problems, promoting social competence: a parent and teacher training partnership in Head Start. J Clin Child Psychol 2001;30:283-301.

20. Gross D, Fogg L, Webster Stratton C, et al. Parent training with multi-ethnic families of toddlers. J Consult Clin Psychol 2003;71:261-78.

21. Sandler IN, Ayers TS, Wolchik SA, et al. The family bereavement program: efficacy evaluation of a theory based prevention program for parents of bereaved children and adolescents. J Consult Clin Psychol 2003;71:587-600.

22. Scott $\mathrm{S}$, O'Connor $\mathrm{T}$, Futh $\mathrm{A}$. What makes parenting programmes work indisadvantaged areas? The PALS trial. A report to the Joseph Rowntree Foundation, 2005.

23. http://www.parentingresearch.org.uk/ (accessed 25 May 2013).

24. Stewart-Brown S, Schrader MacMillan A. Promoting the mental health of children and parents: evidence and outcomes for home and community based parenting support interventions. Report of Workpackage 2 of the DATAPREV Project European Community 6th Framework Research ProgrammeSP5A-CT-2007-044145. http:// wrap.warwick.ac.uk/3239/

25. Webster Stratton C. Parent training with low income clients: promoting parental engagement through a collaborative approach. In: Lutzer JR, ed. Handbook of child abuse research and treatment New York: Plenum Press, 1998:183-210.

26. Webster Stratton C. The Incredible Years: a trouble shooting guide for parents of young children aged 3-8 years. Ontario: Umbrella Press, 2004.

27. Sanders MR, Markie-Dadds C, Turner KMT, et al. Triple $P$ positive parenting programmes: a guide to the system. Brisbane, Australia: Families International, 2000.

28. Sanders MR, Turner KMT, Markie-Dadds C. The development and dissemination of the triple $\mathrm{P}$ positive parenting programme. A multi-level evidence based system of parenting and family support. Prev Sci 2002;3:173-89.

29. Welsh Assembly Government: National Service Framework for Children Young People and Maternity Services in Wales. 2005

30. Welsh Assembly Government: Designed for Life: creating world class health and social care for Wales in the 21st century. 2005.

31. Department of Health. Healthy lives healthy people. London: HM Government, 2010 [cited 10.8.11]. http://www.dh.gov.uk/en/ Publicationsandstatistics/Publications/

PublicationsPolicyAndGuidance/DH 121941

32. Department of Health. No health without mental health: a cross-government mental health outcomes strategy for people of all ages. London: HM Government, 2011 [cited 10.8.11]. http://www.dh. gov.uk/prod consum dh/groups/dh digitalassets/documents/ digitalasset/dh_124058.pdf

33. Department for Education and Skills. Sure start children's centres practice guidance. London: DfES, 2005.

34. Department for Education. Supporting families in the foundation years. London: Department of Education, 2012.

35. Waylen A, Stallard N, Stewart-Brown S. Parenting and health in midchildhood: a longitudinal study. Eur J Public Health 2008;18:300-5.

36. Stewart-Brown S. Public health implications of childhood behaviou problems and parenting programmes. In: Buchanan A, Hudson BL. eds. Parenting, schooling \& children's behaviour: interdisciplinary approaches. Aldershot: Ashgate Publishing, 1998

37. Spoth RL, Kavangh KA, Dishion TJ. Family centred preventive intervention science: toward benefits to larger populations of children, youth and families. Prev Sci 2002;3:145-52.

38. Sanders MR, Cann W, Markie-Dadds C. Why a universal population level approach to the prevention of child abuse is essential. Child Abuse Rev 2003;12:145-54.

39. Heinrichs N, Bertram H, Kuschel A, et al. Parent recruitment and retention in a universal prevention program for child behavior and emotional problems: barriers to research and program participation. Prev Sci 2005;6:275-86.

40. Stewart-Brown S, Anthony R, Wilson L, et al. Should randomized controlled trials be the 'gold standard' for research on preventive interventions for children? J Child Serv 2011;6:228-35.

41. Hiscock H, Bayer JK, Ukoumunne OC, et al. Universal parenting programme to prevent early childhood behavioural problems: cluster randomised trial. BMJ 2008;336:318-21. 
42. Lösel F, Beelmann A, Stemmler M, et al. Prävention von Problemen des Sozialverhaltens im Vorschulalter. [Prevention of social behavior problems at preschool age: evaluation of the parent and child training program package EFFEKT]. ZKlin Psychol Psychother 2006;35:127-39.

43. Sanders MR, Ralph A, Sofronoff K, et al. Every family: a population approach to reducing behavioral and emotional problems in children making the transition to school. J Prim Prev 2008;29:197-222.

44. Prinz RJ, Sanders MR, Shapiro CJ, et al. Population-based prevention of child maltreatment: the U.S. Triple P system population trial. Prev Sci 2009;10:1-12.

45. Zubrick SR, Ward KA, Silburn SR, et al. Prevention of child behaviour problems through universal implementation of a group behavioural family intervention. Prev Sci 2005;6:287-304.

46. Hahlweg K, Heinrichs N, Kuschel A, et al. Therapist assisted self-administered bibliotherapy to enhance parental competence: short and long-term effects. Behav Modif 2008;32:659-81.

47. Kurt $\mathrm{H}$, Heinrichs $\mathrm{N}$, Kuschel $\mathrm{A}$, et al. Long-term outcome of a randomized controlled universal prevention trial through a positive parenting program: is it worth the effort? Child Adolesc Psychiatry Mental Health 2010;4:14-27.

48. Eisner M, Ribeaud D, Juenger R, et al. Fruehpraevention von Gewalt und aggression. In: Ergebnisse des Zuercher Praeventions und Interventions projektes an Schulen [Early prevention of violence and aggression. Results from the Zurich prevention and intervention project at schools]. Zuerich: Rueegger Verlag, 2008:139-83.

49. Hunt C. The parenting puzzle: how to get the best out of family Life. Oxford: Family Links, 2003. http://www.familylinks.org.uk/ nurturing-programme/index.html

50. National Institute for Health and Care Excellence. Antisocial behaviour and conduct disorders in children and young people: recognition, intervention and management. National Institute for Health and Care Excellence, 2013.

51. Barlow J, Stewart-Brown S. Understanding parenting programmes: parents views. Child Care Health Dev 2001;2:117-30.

52. Kirkpatrick S. Family links pilot evaluation study. Oxford: Health Services Research Unit, 2005

53. MacNeill V. The family links nurturing programme: a report of a qualitative evaluation of training and implementation. Oxford: Family Links, 2005.

54. Schultz KF, Altman DG, Moher D. CONSORT 2010 statement: updated guidelines for reporting parallel group randomised trials. BMJ 2010;340:c332.

55. Simkiss DE, Snooks HA, Stallard N, et al. Measuring the impact and costs of a universal group based par enting programme: protocol and implementation of a trial. BMC Public Health 2010;10:364.

56. National Evaluation of Sure Start. The impact of sure start local programmes on child development and family functioning; report of the longitudinal study of 9 month old and 3 year-old children and their families. Institute for the Study of Children, Families and Social Issues, BirkbeckCollege, University of London, 2007.

57. Simkiss DE, MacCallum F, Fan EEY, et al. Validation of the mothers object relations scales in 2-4 year old children and comparison with the child-parent relationship scale. Health Qual Life Outcomes 2013;11:49.

58. Pianta RC. Child-parent relationship scale. Charlottesville, VA: University of Virginia, 1992

59. Caldwell BM, Bradley $\mathrm{RH}$. Home observations for measurement of the environment. Little Rock: University of Arkansas, 1984.

60. Taylor E, Schachar R, Thorley G, et al. Conduct disorder and hyperactivity: 1 . Separation of hyperactivity and antisocial behaviour in British child psychiatric patients. Br J Psychiatry 1986; 149:760-7.

61. Puckering C, Mills M. Mellow parenting-learning to observe parent - child relationships. C\&G Print: Troon, 2004

62. Daley D, Sonuga-Barke EJS, Thompson M. Assessing expressed emotion in mothers of preschool AD/HD children: psychometric properties of a modified speech sample. Br J Clin Psychol 2003;42:53-67.

63. Varni J. Pediatric quality of Life inventory. Version 4. 1998 [cited 26.9.12]. http://www.pedsql.org/pedsql13.htm

64. Tennant R, Fishwick R, Platt S, et al. Monitoring positive mental health in Scotland: validating the Affectometer 2 scale and developing the Warwick-Edinburgh Mental Well-Being Scale. NHS Scotland, 2006

65. Abdin RR. Parenting stress index manual. Charlottesville, VA: Pediatric Psychology Press, 1996.

66. SF-36 User Manual. SF-36 health status questionnaire user's manual. Minneapolis: Quality Quest, 1989.

67. Brazier JE, Roberts J. Estimating a preference-based index from the SF-12. Med Care 2004:42:851-9.

68. Curtis L. ()Unit costs of health and social care. PSSRU: University of Kent, 2009.

69. Efron B. Forcing a sequential experiment to be balanced. Biometrika 1971;58:403-17.

70. Little RJA, Rubin DB. Statistical analysis with missing data. 2nd edn. New York: John Wiley \& Sons, 2002.

71. O'Neil D, McGilloway S, Donnelly M, et al. A cost effectiveness analysis of the Incredible Years parenting programme in reducing childhood health inequalities. Eur J Health Econ 2013;14:85-94.

72. Little M, Berry V, Morpeth L, et al. The impact of three evidencebased programmes delivered in public systems in Birmingham, UK. Int J Confl Violence 2012;6:262-72.

73. HM Revenue \& Customs approved mileage rates 2002/03 to 2010/ 11 http://www.hmrc.gov.uk/rates/travel.htm 